\title{
The effect of volume fraction concentration on the thermal conductivity and thermal diffusivity of nanofluids: numerical and experimental.
}

\begin{abstract}
This article reports on the effect of aluminum (Al) volume fraction concentration on the thermal conductivity and thermal diffusivity of Al nanoparticles suspended in water, ethylene glycol, and ethanol based fluids prepared by the one step method. The Al nanoparticles were independently produced and then mixed with a base fluid to produce the nanoparticles suspension. The thermal conductivity and thermal diffusivity of the nanofluids were measured using the hot wire-laser beam displacement technique. The thermal conductivity and thermal diffusivity were obtained by fitting the experimental data to the numerical data simulated for $\mathrm{Al}$ in distilled water, ethylene glycol, and ethanol. The thermal conductivity and thermal diffusivity of the nanofluids increase with an increase in the volume fraction concentration.
\end{abstract}

Keyword: Volume fraction concentration; Thermal conductivity; Thermal diffusivity; Nanofluids 